${ }^{1}$ Department of Orthodontics and Radiology, UNICID (University of São Paulo City), São Paulo, $\mathrm{SP} /$ Brazil

${ }^{2}$ Neuroimaging Laboratory, Department of Neurology, UNICAMP (University of Campinas) Campinas - SP/Brazil.
Corresponding author:

Ana Carla Raphaelli Nahás-Scocate, Department of Orthodontics and Radiology, UNICID, Rua Cesário Galeno 448, Bloco A. Tatuapé, São Paulo, SP- CEP 03071-000 Email: carlanahas@yahoo.com.br

Received: June 6, 2017

Accepted: July 26, 2017

\section{Evaluation of the Skeletal Maturation of Cervical Vertebrae with Magnetic Resonance Imaging: a piloty study}

Camila Chaparin Baldin ${ }^{1}$, DDS, M.Sc; Marjorie Kitt ${ }^{1}$, UGS; André Luiz Ferreira Costa ${ }^{1,2}$, DDS, PhD; Clarissa Lin Yasuda², MD, PhD; Fernando Cendes², MD, PhD; Ana Carla Raphaelli Nahás-Scocate ${ }^{1}$, DDS, PhD

Aims: The objective of the present study was to assess the skeletal maturation by means of three-dimensional models of the cervical vertebrae generated through segmentation of the magnetic resonance (MR) images by using medical software. Methods: Twenty MR images of the skull of male and female individuals aged between 8 and 22 years old were selected. Assessment of the images was performed by using the ITK-SNAP software, consisting of three steps: 1) vertebral segmentation; 2) three-dimensional reconstruction; and 3) classification of skeletal maturation. Two specialists in orthodontics and two specialists in dentomaxillofacial radiology assessed the images. Results: Analysis of reproducibility and repeatability were performed by using the RR method, with paired t-test also being applied to the repeatability factor together with Lin's concordance coefficient. The significance level was set at $5 \%$. It was found that there was no difference in the inter-rater reliability $(P$-value $=0.625)$, but without statistical repeatability. Conclusions: New tools, as $3 \mathrm{D}$ reconstruction software, enabled us to build an effective and friendly 3D-reconstruction system for classification of the skeletal maturation stages of cervical vertebrae.

Keywords: Maturation; cervical vertebrae; magnetic resonance image; $3 \mathrm{D}$ reconstruction 


\section{Introduction}

In medicine and dentistry it is fundamental to determine the growth process of an individual in order to know the related disorders in endocrinology and orthopaedics, as well as to assist in the diagnosis and planning of the orthodontic treatment ${ }^{1,2}$. This growth process has a great variability from individual to individual, being influenced by several factors ${ }^{3}$.

The term "skeletal maturation age" was introduced in order to analyse and differentiate with greater accuracy the biological age, skeletal age and skeletal maturation found in the same individual ${ }^{4}$. There are different forms of estimating the maturational process. However, despite the reliability of the different forms of assessment, the one regarding skeletal maturation is the most reliable and efficient, since the variations in the form and density of the bone allow to gauge the growth trajectory during development ${ }^{5}$.

The study of skeletal maturation is performed by means of radiographs, which serve to evaluate ossification centres based on the emergence or absence of certain structures and development of others ${ }^{6}$. With this aim in mind, radiographs of various parts of the human body are used, with hand and wrist bones being the most useful and recognised $^{7-9}$. Despite being a simple and cheap method, there is a great inconvenience in using this method because of the extra ionising radiation to the patient ${ }^{10}$.

Nowadays, there is an increasing tendency to use other structures to estimate the skeletal age, such as cervical vertebrae visualised on lateral teleradiographs. Studies by Franchi et al. ${ }^{11}$ and Baccetti et al. ${ }^{12}$ corroborate that this is an adequate method for assessing the skeletal maturation as they found a positive relationship between cervical vertebrae and mandibular growth during puberty. In this methodology, the stages representing different morphological characteristics of the cervical vertebrae are defined, with each of these stages reflecting the progressive levels of skeletal maturation ${ }^{4}$. However, the method is considered subjective, since it is based on visual exams of the morphological changes in the cervical vertebrae. Additionally, the evaluator is required to have experience in order to establish a correct diagnosis ${ }^{13,14}$.

According to Mendelson and Rubin ${ }^{15}$, the advances in the computing field allows for the development of better software packages. Therefore, these software packages are of great value for radiological diagnosis ${ }^{16}$.

There are currently software packages enabling both visualisation and segmentation of images from magnetic resonance (MR) image and computed tomography (CT), including rendering and classification of 3D models ${ }^{17-21}$. This process provides important information both didactically and diagnostically, allowing better localisation of structures, surgical planning and exam analysis ${ }^{22}$.

There is no study in the literature showing assessment of cervical vertebrae by means of MR imaging. Therefore, we have proposed to develop and implement a semi-automatic methodology for assessing the skeletal maturation of cervical vertebrae from MR images of the skull by using free software. 


\section{Material \& Methods}

This study was approved by the local Research Ethics Committee according to protocol number 999.190. The images were originally taken as part of previous MR images of the central nerve system and were obtained from the Laboratory of Neuroimaging of School of Medicine of University of Campinas (UNICAMP). There was no need to recruit further subjects for conduction of MR exam. Only images of subjects who had no disease which might result in oromaxillofacial changes were included for study. Twenty images of male and female individuals comprised the sample, which was divided into four groups of five subjects each: G1 (8-10 years old); G2 (12-14 years old); G3 (16-18 years old); and G4 (20-22 years old). The inclusion criteria were the following: MR images of the skull of individuals aged between 8 and 22 years old. On the other hand, the exclusion criteria were: history of facial injury due to accidents; previous orthodontic treatment; and any pathology making interpretation of the results difficult.

The MR images were obtained by using a 3-Tesla scanner (Phillips Achieva-Intera). The image acquisition parameters were: spin echo T1 weighted sagittal images (6-mm thickness, tip angle of $180^{\circ}, \mathrm{TR}=430, \mathrm{TE}=12,200 \times 350$ matrix, and FOV $=25 \times 25 \mathrm{~cm}$ ). These images were used to guide the acquisition plane for axial and coronal images.

The MR images were acquired in ANALYZE format and then converted into DICOM format by using the MIPAV software (mipav.cit.nih.gov). The ITK-SNAP software (www.itksnap.org) ${ }^{23}$ was used to perform the image segmentation and to obtain 3D models. This software also allows for semi-automatic segmentation in which several colours can be chosen for the structures delineated. In the segmentation, the similarity in the shades of gray among equal-density tissues allows to locate image edges and consequently the total mapping of the structure being studied ${ }^{24}$.

For obtaining the 3D models from each MR image, 10 slices (84 to 94) were selected before thresholding of each image of the vertebrae (i.e. C2, C3, C4). After delineation, the vertebrae were filled with the selected colours. In the end of this process, the models were generated for analysis.

The method used in this work was the interactive thresholding by manipulating the image histogram. This technique is based on determining two values termed minimum and maximum thresholds, which delineate in the histogram the region of interest (ROI) to be segmented. The segmentation was performed by grouping neighbour pixels with similar values at a tolerance level. Next, the segmented image is extracted from other surrounding structures, reconstructed and visualised three-dimensionally ${ }^{17,24}$.

Our regions of interest were the cervical vertebrae C2, C3 and C4. The software threshold was used for segmentation of the images based on defining density intervals expressing, for example, only voxels which correspond to the desired cervical vertebra (Fig. 1a). For doing so, a detection algorithm is applied to each transversal slice to define each contour. After some tests with several detection algorithms, we found the most satisfactory one for the purposes of this study. Each object was assigned a label for identification.

After mapping the vertebra, the 3D reconstruction of the object was performed (Fig. 1b). Image assessment was conducted by two orthodontists and two oral radiologists, previously calibrated, who used supporting material containing skeletal maturation stages 

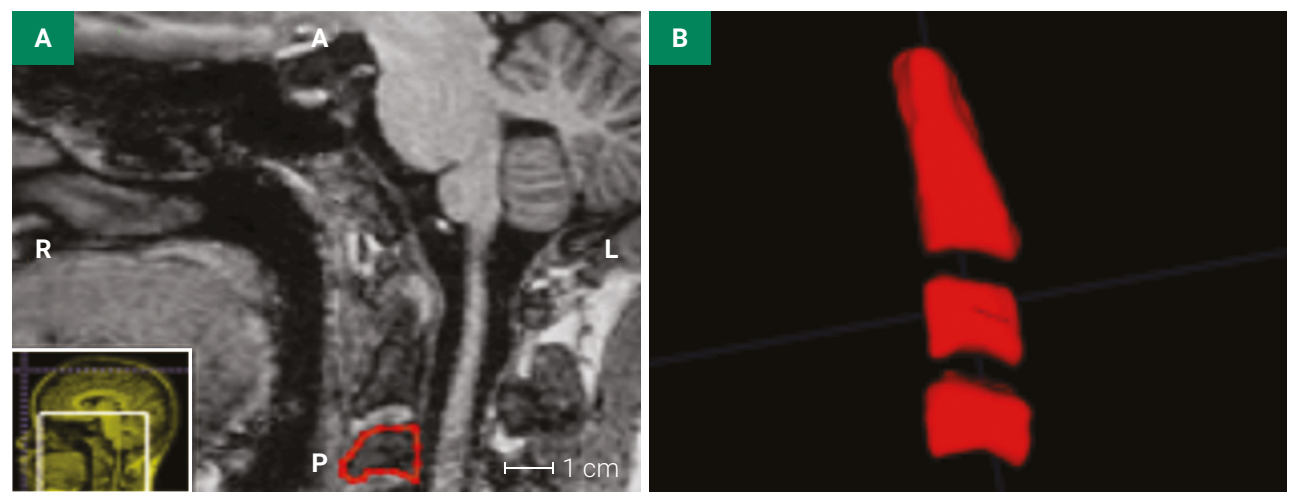

Figure 1. a) Segmentation of vertebra C3 by using the software ITK-SNAP; b) 3D model of vertebrae C2, $\mathrm{C} 3$ and $\mathrm{C} 4$ generated by segmentation.

and description of each stage as suggested by Hassel \& Farman (1995). The evaluators analysed and evaluated the twenty 3D images and then answered a 3-item questionnaire on the method used. They re-evaluated the images after 1-week interval.

Exploratory analysis of the data was carried out based on resumed measurements (i.e. mean, standard deviation, minimum, median, maximum values) and graph construction. Analysis of repeatability and reproducibility of the method was performed by using the RR method. For assessment of repeatability, the paired t-test for each evaluator and Li's concordance coefficient were used at significance level of $5 \%$ by using the Minitab statistical software, version 16 R302.

\section{Results}

Table 1 shows the measurements of position and dispersion per evaluator and repetition; the elaboration and conduction of the study on repeatability and reproducibility of the method.

In the graph of variation components, the contribution percentage of the patients is higher than that of study, demonstrating that the largest part of the variation found in the study is due to differences between patients, but more than $30 \%$ (63.02) of the variation is due to measurement system (Fig. 2).

Table 1. Measurements of position and dispersion of skeletal maturation per evaluator and repetition.

\begin{tabular}{cccccccc}
\hline Evaluater & Repetition & N & Mean & SD & Minimum & Median & Maximum \\
\hline 1 & 1 & 20 & 3.2 & 1.2 & 1.0 & 3.5 & 5.0 \\
\hline 1 & 2 & 20 & 3.5 & 1.3 & 2.0 & 3.5 & 6.0 \\
\hline 2 & 1 & 20 & 2.8 & 1.7 & 1.0 & 2.0 & 6.0 \\
\hline 2 & 2 & 20 & 3.4 & 1.9 & 1.0 & 3.0 & 6.0 \\
\hline 3 & 1 & 20 & 3.0 & 1.3 & 1.0 & 3.0 & 5.0 \\
\hline 3 & 2 & 20 & 3.7 & 1.2 & 2.0 & 3.0 & 6.0 \\
\hline 4 & 1 & 20 & 3.3 & 1.4 & 1.0 & 3.5 & 5.0 \\
\hline 4 & 2 & 20 & 3.2 & 1.4 & 1.0 & 3.0 & 6.0 \\
\hline
\end{tabular}




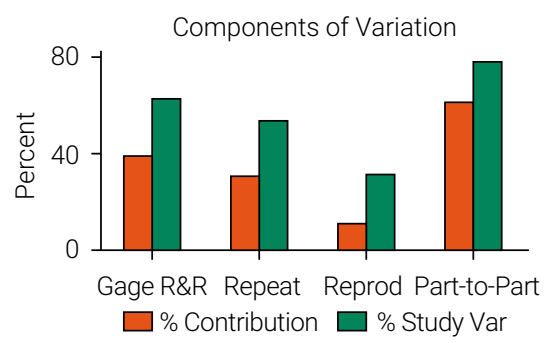

R Chart by Evaluatot

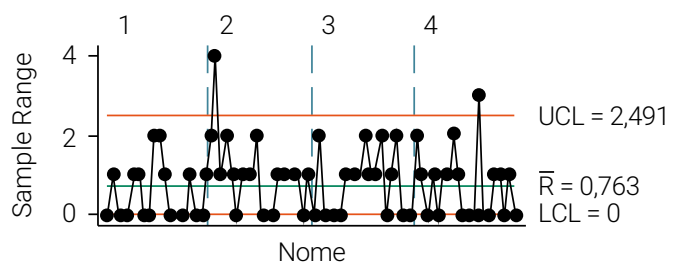

Nome

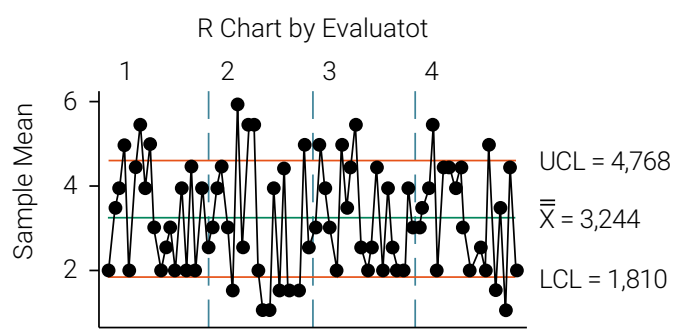

Maturation by patient

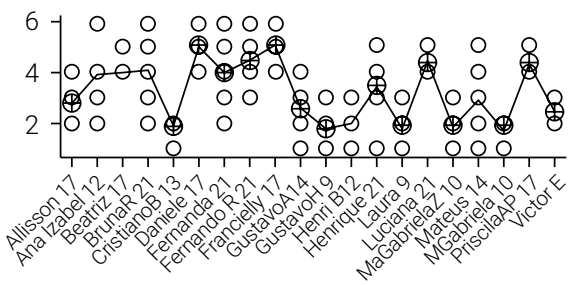

Maturation by Evaluator

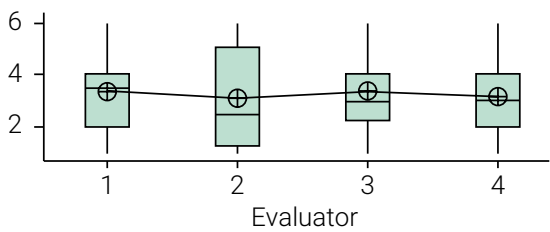

Patient * Evaluator Interaction

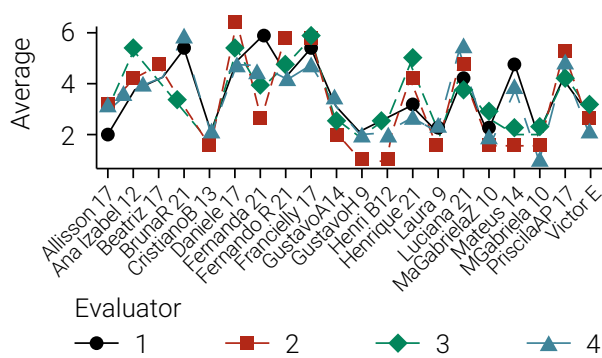

Figure 2. Study of repeatability and reproducibility of the method.

In the graph per patient, there are differences between patients as expected and shown by the non-linear straight line linking their mean values. In the graph per evaluator, there is a small difference between the evaluators as also expected and shown by the almost non-linear straight line linking their mean values (Fig. 2).

In the $x$-bar graph per evaluator, the majority of the points are within the limits, indicating a variation in the measurement system. The graph evaluator*patient shows an interaction between these two variables $(P$-value $=0.017)$, indicating that there was a difference in the process of assessment made by each evaluator regarding each patient (Fig. 2).

It was found that there was no difference between the evaluators ( $P$-value $=0.625)$, despite the poor repeatability. Paired t-test was performed for each evaluator in order to compare their answers between the repetitions. No statistically significant differences were found between the repetitions performed by evaluators 1 $(P$-value $=0.110)$ and $4(P$-value $=0.847)$. There was found no statistically significant difference between the two repetitions performed by evaluator $2(P$-value $=0.077)$. On the other hand, a significant difference was observed between the repetitions performed by evaluator $3(P$-value $=0.004)$. The Lin's concordance coefficient showed around a coefficient of 0.7 . 


\section{Discussion}

Hand-wrist radiograph has been used in the orthodontic practice for assessment of the ossification centres, as it is possible to find distinct modifications in each one during their development. These changes are used to inform the orthodontist about the period of skeletal maturation in which the patient is ${ }^{7-9}$. Fishman ${ }^{7}$ observed a synchronism in the growth of several body structures, showing that the information on hand and wrist structures can be used for assessing the general growth of the body and face as well1,10,25.

Although the hand-wrist radiograph is consecrated in the orthodontic practice, there is currently a trend towards the use of alternative methods for determination of the growth spurt, such as the observation of cervical vertebrae ${ }^{6,8,26}$. According to Hassel and Farman ${ }^{4}$, the cervical vertebrae are viewed on lateral teleradiographs comprising the routine documentation of patients who are submitted to orthodontic and/or functional orthopaedic treatment, which can reduce the amount of ionising radiation to the patient ${ }^{9,13}$.

The efficacy in using cervical vertebrae by means of lateral teleradiography was also evaluated by Wong et al. ${ }^{1}$, Kucukkles et al. ${ }^{6}$, Flores et al. ${ }^{8}$, Danaei et al. ${ }^{9}$, Caldas et al. ${ }^{13}$, Gandini et al. ${ }^{25}$ and Mahajan ${ }^{27}$, who concluded that this alternative method is a reliable parameter to assess the skeletal maturation as it helps to determine the best time to initiate the orthopaedic intervention in patients in the stage of craniofacial growth. The range of information provided by the assessment of cervical vertebrae can replace the traditional method based on hand-wrist radiographs, thus simplifying the orthodontic routines the patients face and preventing additional dose of ionising radiation to them.

Assessing the cervical vertebral maturation using MR images is suggested by the present study, since there is no work in the literature relating visualisation methods to interpretation of the skeletal maturation. We consider that our work is a pilot study because it addresses an innovative method in the dentistry field, favouring the patients as they are less exposed to ionising radiation. However, it is natural that many biases emerge during the development of a study method.

According to the results found, it is suggested that the number of evaluators should be higher in further studies and that they should be rigorously trained, since it is known that assessment of 3D models requires a qualified evaluator. On the other hand, this method is easy and quick to understand and this has encouraged us to improve it in further studies. The fact that the evaluator can also manipulate the image of the vertebrae by using software makes this method dynamic and non-static.

The method of assessing the cervical vertebrae by means of magnetic resonance imaging, as suggested in our study, has innovated the dental and medical scopes scientifically as the patient is less exposed to radiation. Demystifying the use of 3D image in the final diagnosis is part of our ongoing achievement for advance of imagenology.

\section{Conclusion}

According to the methodology applied and the results obtained, one can conclude that there was no difference between the evaluators despite the poor repeatability. The method's reproducibility depends on systematisation of the measurements, since 
the assessment of 3D images requires well-trained evaluators. The skeletal maturation stages of the cervical vertebrae followed the same pattern, suggesting that this proposed methodology deserves to be improved as no ionising radiation is involved.

\section{Acknowledgements}

This work was supported by São Paulo Research Foundation - FAPESP (grant number 12/16953-4).

\section{Declaration of Conflicting Interests}

The Authors declare that there is no conflict of interest.

\section{References}

1. Wong RW, Alkhal HA, Rabie AB. Use of cervical vertebral maturation to determine skeletal age. Am J Orthod Dentofacial Orthop. 2009 Oct;136(4):484.e1-6; discussion 484-5. doi: 10.1016/j.ajodo.2007.08.033.

2. Crawford B, Kim DG, Moon ES, Johnson E, Fields HW, Palomo JM, et al. Cervical vertebral bone mineral density changes in adolescents during orthodontic treatment. Am J Orthod Dentofacial Orthop. 2014 Aug;146(2):183-9. doi: 10.1016/j.ajodo.2014.04.019.

3. Linhares RV, Matta Mde O, Lima JR, Dantas PM, Costa MB, Fernandes Filho J. [Effects of sexual maturation on body composition, dermatoglyphics, somatotype and basic physical qualities of adolescents]. Arq Bras Endocrinol Metabol. 2009;53(1):47-54. portuguese.

4. Hassel B, Farman AG. Skeletal maturation evaluation using cervical vertebrae. Am J Orthod Dentofacial Orthop. 1995 Jan;107(1):58-66

5. Baccetti T, Franchi L, De Toffol L, Ghiozzi B, Cozza P. The diagnostic performance of chronologic age in the assessment of skeletal maturity. Prog Orthod. 2006;7(2):176-88.

6. Kucukkeles N, Acar A, Biren S, Arun T. Comparisons between cervical vertebrae and hand-wrist maturation for the assessment of skeletal maturity. J Clin Pediatr Dent. 1999 Fall;24(1):47-52.

7. Fishman LS. Radiographic evaluation of skeletal maturation. A clinically oriented method based on hand-wrist films. Angle Orthod. 1982 Apr;52(2):88-112.

8. Flores-Mir C, Burgess CA, Champney M, Jensen RJ, Pitcher MR, Major PW. Correlation of skeletal maturation stages determined by cervical vertebrae and hand-wrist evaluations. Angle Orthod. 2006 Jan; 76(1):1-5.

9. Danaei SM, Karamifar A, Sardarian A, Shahidi S, Karamifar H, Alipour A, et al. Measuring agreement between cervical vertebrae and hand-wrist maturation in determining skeletal age: reassessing the theory in patients with short stature. Am J Orthod Dentofacial Orthop. 2014 Sep;146(3):294-8. doi: 10.1016/j.ajodo.2014.05.023.

10. Santiago RC, Cunha AR, Junior GC, Fernandes N, Campos MJ, Costa LF, et al. New software for cervical vertebral geometry assessment and its relationship to skeletal maturation--a pilot study. Dentomaxillofac Radiol. 2014;43(2):20130238. doi: 10.1259/dmfr.20130238.

11. Franchi L, Baccetti T, McNamara JA, Jr. Mandibular growth as related to cervical vertebral maturation and body height. Am J Orthod Dentofacial Orthop. 2000 Sep;118(3):335-40.

12. Baccetti T, Franchi L, McNamara JA, Jr. An improved version of the cervical vertebral maturation (CVM) method for the assessment of mandibular growth. Angle Orthod. 2002 Aug;72(4):316-23. 
13. Caldas Mde P, Ambrosano GM, Haiter Neto F. New formula to objectively evaluate skeletal maturation using lateral cephalometric radiographs. Braz Oral Res. 2007 Oct-Dec;21(4):330-5.

14. Nestman TS, Marshall SD, Qian F, Holton N, Franciscus RG, Southard TE. Cervical vertebrae maturation method morphologic criteria: poor reproducibility. Am J Orthod Dentofacial Orthop. 2011 Aug;140(2):182-8. doi: 10.1016/j.ajodo.2011.04.013.

15. Mendelson DS, Rubin DL. Imaging informatics: essential tools for the delivery of imaging services. Acad Radiol. 2013 Oct;20(10):1195-212. doi: 10.1016/j.acra.2013.07.006.

16. Doi K. Computer-aided diagnosis in medical imaging: historical review, current status and future potential. Comput Med Imaging Graph. 2007 Jun-Jul;31(4-5):198-211.

17. Costa AL, Yasuda CL, Appenzeller S, Lopes SL, Cendes F. Comparison of conventional MRI and 3D reconstruction model for evaluation of temporomandibular joint. Surg Radiol Anat. 2008 Nov:30(8):663-7. doi: 10.1007/s00276-008-0400-z.

18. Chang G, Honig S, Brown R, Deniz CM, Egol KA, Babb JS, et al. Finite element analysis applied to 3-T MR imaging of proximal femur microarchitecture: lower bone strength in patients with fragility fractures compared with control subjects. Radiology. 2014 Aug;272(2):464-74 doi: 10.1148/radiol.14131926.

19. Cevidanes LH, Gomes LR, Jung BT, Gomes MR, Ruellas AC, Goncalves JR, et al. 3D superimposition and understanding temporomandibular joint arthritis. Orthod Craniofac Res. 2015 Apr;18 Suppl 1:18-28. doi: 10.1111/ocr.12070.

20. Schilling J, Gomes LC, Benavides E, Nguyen T, Paniagua B, Styner M, et al. Regional 3D superimposition to assess temporomandibular joint condylar morphology. Dentomaxillofac Radiol. 2014;43(1):20130273. doi: 10.1259/dmfr.20130273.

21. Alhadidi A, Cevidanes LH, Paniagua B, Cook R, Festy F, Tyndall D. 3D quantification of mandibular asymmetry using the SPHARM-PDM tool box. Int J Comput Assist Radiol Surg. 2012 Mar;7(2):265-71. doi: 10.1007/s11548-011-0665-2.

22. White SC. Computer-aided differential diagnosis of oral radiographic lesions. Dentomaxillofac Radiol. 1989 May; 18(2):53-9

23. Yushkevich PA, Piven J, Hazlett HC, Smith RG, Ho S, Gee JC, et al. User-guided 3D active contour segmentation of anatomical structures: significantly improved efficiency and reliability. Neuroimage. 2006;31(3):1116-8.

24. Yasuda CL, Costa AL, Franca M, Jr., Pereira FR, Tedeschi H, de Oliveira E, et al. Postcraniotomy temporalis muscle atrophy: a clinical, magnetic resonance imaging volumetry and electromyographic investigation. J Orofac Pain. 2010 Fall;24(4):391-7.

25. Gandini P, Mancini M, Andreani F. A comparison of hand-wrist bone and cervical vertebral analyses in measuring skeletal maturation. Angle Orthod. 2006 Nov;76(6):984-9.

26. Patcas R, Signorelli L, Peltomaki T, Schatzle M. Is the use of the cervical vertebrae maturation method justified to determine skeletal age? A comparison of radiation dose of two strategies for skeletal age estimation. Eur J Orthod. 2013 Oct;35(5):604-9. doi: 10.1093/ejo/cjs043.

27. Mahajan S. Evaluation of skeletal maturation by comparing the hand wrist radiograph and cervical vertebrae as seen in lateral cephalogram. Indian J Dent Res. 2011 Mar-Apr;22(2):309-16. doi: 10.4103/0970-9290.84310. 